This item was submitted to Loughborough's Research Repository by the author.

Items in Figshare are protected by copyright, with all rights reserved, unless otherwise indicated.

\title{
A fog caching scheme enabled by ICN for loT environments
}

PLEASE CITE THE PUBLISHED VERSION

https://doi.org/10.1016/j.future.2020.04.040

PUBLISHER

Elsevier

VERSION

AM (Accepted Manuscript)

PUBLISHER STATEMENT

This paper was accepted for publication in the journal Future Generation Computer Systems and the definitive published version is available at https://doi.org/10.1016/j.future.2020.04.040.

\section{LICENCE}

CC BY-NC-ND 4.0

\section{REPOSITORY RECORD}

Hua, Yining, Lin Guan, and Kostas Kyriakopoulos. 2020. "A Fog Caching Scheme Enabled by ICN for lot Environments". Loughborough University. https://hdl.handle.net/2134/12191847.v1. 


\title{
A Fog Caching Scheme Enabled by ICN for IoT Environments
}

\author{
Yining Hua ${ }^{\mathrm{a}}$, Lin Guan ${ }^{\mathrm{a}, *}$, Konstantinos Kyriakopoulos ${ }^{\mathrm{b}}$ \\ ${ }^{a}$ Department of Computer Science, Loughborough University, Loughborough, UK \\ ${ }^{b}$ Wolfson School of Mechanical, Electrical and Manufacturing Engineering, Loughborough University, \\ Loughborough, UK
}

\begin{abstract}
The rapid growth in computation and processing power of end-user devices has transitioned network functionalities from the core network to the Fog, thus, reducing response times and ultimately improving user experience. The Information-Centric Networking paradigm aims to transform conventional content caching and delivery approaches by enabling Fog nodes to participate in both forwarding and caching. In this work, a Fog caching design scheme is presented for applications such as Internet-of-Things, which integrates three novel design attributes. Firstly, a Fog cluster-based scheme is proposed that utilises both in-network and end-user devices to cache content closer to the edge network, according to the increasing popularity of the content. Secondly, this work proposes a near-path approach that leverages caching nodes near the content delivery path. Finally, by craftily integrating reactive and proactive caching, congestion during network peak-time is averted. Simulations are conducted in the Icarus environment and evaluated against eight popular benchmark techniques. The results indicate significant improvement in internal link load and path stretch metrics. Finally, the cache hit ratio metric is consistently better than all other benchmarks, while the latency performance of the proposed scheme is competitive when the content distribution is more concentrated. Keywords: In-network Caching; Information-Centric Network; Peer-to-Peer Cache; User Generated Content; Content Delivery; Content Placement Strategy.
\end{abstract}

\footnotetext{
*Corresponding author

Email addresses: Y.Hua@lboro.ac.uk (Yining Hua), L.Guan@lboro.ac.uk (Lin Guan), K.Kyriakopoulos@lboro.ac.uk (Konstantinos Kyriakopoulos)
}

Preprint submitted to Special Issue on "Information-Centric Network enabler Communication for Internet of Things"April 24, 2020 


\section{Introduction}

Internet-of-Things (IoT) is gradually establishing itself as a driving force for technological advancement creating impact in multiple sectors, including smart homes and cities, intelligent transport systems, industrial control systems and even e-health ser5 vices. Despite the new technological opportunities, a number of IoT related challenges arise. In particular, the security of sensitive IoT data is paramount due to privacy concerns [1]. In addition, the sheer volume of IoT traffic and the immediate response time expected from IoT services mandate novel approaches towards load balancing and delay tolerance [2].

Information-Centric Networking (ICN), a next generation network architecture, adopts a content-centric approach that could be used for IoT content delivery, whilst abiding to security related requirements [3]. The inherent in-network caching capability of ICN also has the potential to significantly reduce the traffic load and transmission delay in next-generation networks [4, 5].

With the rapid growth of computational and storage capabilities in IoT devices, network functions, such as content delivery and load balancing, could transition from the network core to the Fog. To this end, there is an increasing research momentum for utilising the ICN architecture to address challenges in Fog networks. The authors in [6] present an overview of the challenges and development road-map for Data-Centric nature of Fog networks, by using qualifiers that Fog nodes must present in order to receive data in an ICN paradigm. Furthermore, to extend the conventional Fog-toCloud communication paradigm, the authors in [8] introduce a horizontal Fog-to-Fog communication layer enabled through ICN.

In a similar spirit, this work aims to design a Fog-caching scheme for IoT applications leveraging the ICN architecture. Specifically, according to our comprehensive investigation, as described in the next section, six caching design drivers are identified in existing in-network caching approaches. Conventional works in this area are focusing on a subset of the these drivers. To incorporate all six drivers, our proposed scheme design meets three unique attributes, as follows. 
Firstly, the caching nodes within the network Fog compose one or more adjustable sets, Fog-Clusters ( $F C$ s), each serving a group of users for a fixed period of time. During the caching process, the requested contents are initially cached into the Fog's highest tier caches. As the content gets increasingly requested, it is gradually cached in lower tiers, i.e. closer to the user side. In order to adjust to topology changes and utilise potential available storage resources, both in-network and end-user caching devices, the cluster border can periodically be extended towards upper and/or lower tiers.

Secondly, instead of traditional on-path and off-path caching [9], caching nodes near the delivery path are also considered as elements in the $F C$. In this way, the commonly demanded content can be cached close to multiple delivery paths and increase the caching node utilisation.

Finally, as an integration of reactive and proactive caching, full-time caching is introduced to reduce delivery delay and peak-time traffic load. In full-time caching, reactive caching is triggered only once, when the content is delivered from its original ${ }_{45}$ provider to the end-user. Thus, at least one content could be cached in Fog caching nodes. Proactive caching is only triggered in off-peak hours to populate Fog caching nodes without incurring excessive traffic during peak-time content delivery.

The original contribution of the paper is summarised as follows:

- A novel caching scheme is proposed that incorporates six identified drivers through comprehensive investigation of conventional in-network caching schemes. Specifically, the proposed scheme has three outstanding attributes: 1) flexibility achieved through periodically adjustable clusters 2) near-path content allocation utilising node near the delivery path and 3) full-time caching, combining proactive and reactive approaches.

- The proposed caching system is modelled in two parts. Initially, set theory is applied to assign permanent characters, which represent the forwarding and storage capabilities of each node. In addition, temporary roles dictated by logical coordinates are allocated to identify the cluster and tier of involved caching nodes during a particular content request session.

- Simulated experiments are designed to model a dense Fog caching environment 
for IoT applications. In our experimental design, both end-devices and ICN innetwork nodes are configured for caching purposes. Moreover, the dependence on the backbone network is limited and emphasis is placed on the network Fog. To design scenarios of mixed request traffic, a number of end devices are nominated as data sources for both user-generated content and content-on-demand.

- The proposed scheme achieves better performance in four evaluation metrics; latency, cache hit ratio, internal link load and path stretch. Evaluation and analysis is conducted against popular schemes i.e. Edge Caching, Consumer Caching, Leave Copy Down [10], Leave Copy Everywhere, Random Choice [11], Random Bernoulli, [12], Cache Less for More [13] and ProbCache [14].

The rest of this paper is structured as follows. Section 2 summarises the state-of-the-art ICN Fog caching schemes for IoT applications according to six design drivers. The proposed system design is presented in Section 3 , where three attributes that satisfy the design drivers are illustrated. Section 4 describes the proposed system model and describe how permanent characters and temporary roles are allocated to Fog nodes. Section 5 provides the algorithmic implementation of the proposed scheme. The experimental setting, including parameter configuration, topology design and performance metrics are described in Section 6 , followed by the result discussion in Section 7. Finally, the paper concludes in Section 8 with a discussion on future work.

\section{Related Work}

With the rapid development of IoT technologies, the research emphasis of ICN architectures is moving towards the network Fog and Edge [15]. For example, some researchers combine the Named Data Network (NDN) architecture with Edge computing to meet the IoT requirements [16]. In addition, others suggest to apply deep-learning intelligence on the ICN-IoT platform at the network Edge to provide better services [17]. However, since the ICN concept was firstly proposed in the TRIAD project [18], the multiple naming and resolution design push the ICN approaches into several distinct directions [19]. For instance, some ICN architecture, such as NDN [20] adopts 
hierarchical naming or naming schemes with attribute-based features [21], while others, such as DONA [22] and PURSUIT [23], select flat naming method in their design [24]. Nevertheless, in-network caching is an essential and common component in ICN architectures, especially at the network Fog.

In the following paragraphs, the conventional in-network caching literature can be categorised according to six design drivers.

95 1. Caching Granularity: Regarding the granularity of cached content, the existing schemes have attempted to store the content in packets, chunks or object units. The typical packet-level caching scheme was initially proposed by the authors in [25] and explores the feasibility of functional network-level packet caches in ICN routers. For chunk-level caching, WAVE [26] splits content into chunks, and contents popularity is used to determine the number of cached chunks for specific content. In addition, coded caching [27] also splits the content into chunks and caches the chunks for the same content in different caching nodes. According to the authors in [28], coded caching can achieve better performance than uncoded schemes in broadband multi-media services, such as video-on-demand (VoD). However, in most IoT applications, the contents are not only on-demand but also generated by end-users. Thus, considering such IoT traffic patterns, object level caching is better suited to the design characteristics of the proposed IoT-oriented scheme, due to the lower delay tolerance, smaller content size, larger number of contents and shorter expiration time in User Generated Content [29]. 2. Caching Node Placement: Conventional literature in caching node placement could be divided into ubiquitous caching, Fog caching and Edge caching. Compared to ubiquitous caching with high management cost [30] and Edge caching with restricted end-device capacity [31], the Fog placement is emerging as an ideal caching position for some applications [32]. Moreover, adjustable Fog caching, which can work together with end-user caching devices and conventional storage servers, is motivated by the intrinsically hierarchical "Cloud-Fog-Edge" network architecture [33]. Thus, our proposed scheme focuses on adjustable and distributed Fog-caches incorporating end-user and in-network caching nodes.

3. Storage Capacity Allocation To explore the efficient storage allocation, the authors in [34] compared heterogeneous allocation with homogeneous cache size distribution. 
Their result indicated that the improvement of heterogeneous allocation based on topological graph properties is very limited. Thus, the storage capacity allocation in the proposed scheme chooses a homogeneous distribution, meaning that the caching capacity of every caching node is uniform.

4. Caching Timing: Caching timing determines when the caching operation is triggered. In conventional schemes, the caching timing may be either reactive or proactive [35]. Reactive caching means the caching operation is only triggered during the content delivery session [36]. On the other hand, proactive caching schemes populate the caching nodes regularly but outside the delivery session (e.g. during off-peak hours) [37]. In this paper, a full-time scheme is introduced as a combination for reactive and proactive caching.

5. Content Eviction: Once caching nodes get full, there is a need for content eviction schemes to release the caching space for the new coming contents. So far, most of the conventional content eviction algorithms are derived from the cache replacement ones for operational system caching. Aiming at the ideal Belady's MIN [38], basic policies (i.e. First-In-First-Out (FIFO), Least Frequently Used (LFU), Least Recently Used (LRU)) based on content freshness, content request frequency and request recentness are used for content eviction purposes. Since almost all the conventional caching schemes use LRU, a line speed scheme, in their implementation, the proposed scheme also uses LRU to maintain an fairness in evaluation.

140 6. Content Placement: Content placement is the most active research topic and is responsible for deciding which nodes will cache a particular content. Based on whether contents are placed into caching nodes on or out of the delivery path, conventional caching schemes are categorised into on-path or off-path. Most of the existing schemes in the literature are on-path schemes. For example, Leave Copy Everywhere (LCE), random probabilistic caching (RND), UniCache, Random Insertion (RAND) and Leave Copy Down (LCD) [10] are typical on-path content placement strategies [39].

LCE caches the content in every single caching node along the delivery path. In order to reduce LCE's excessive caching, RND and UniCache introduce a caching probability. In RND, the content is cached into each on-path caching node following a randomly generated probability. UniCache uses a fixed probability equal to the inverse 
of the hop-distance of the delivery path and, as a result, caches a specific requested content only in a single location along the delivery path. Similarly, LCD and RAND are two other caching strategies, which cache a content once along each delivery path. Under the LCD strategy, the content's caching position is moved towards the end-users with increasing number of requests. In RAND, a random node on the delivery path is selected to cache the content. Besides the traditional strategies mentioned above, some more comprehensive content placement strategies have been proposed according to different criteria, such as content popularity and probability.

WAVE [26] is a popularity-based strategy, in which the content is split into chunks during the caching process. The number of cached content chunks in each caching node is exponentially increased along with the increasing number of request. In addition, ProbCache is another probability-based strategy [14], in which the probability of caching is calculated and stored in every caching node depending on several factors, such as hop distance from source and caching space remaining on the path. Furthermore, Popularity based Probability Caching [40] is based on both content probability and popularity and can enhance the content delivery services in named data networks.

Furthermore, the graph properties of a network topology could also be used to decide on content placement. For example, Cache Less for More (CL4M) selects the betweenness centrality graph property. When the content is delivered towards the user, 170 a content is left into the caching node with the highest betweenness centrality value. The criteria for content placement decision could also be a combination of topological information and content characteristics [13]. For example, Age-based Cooperative Caching (ABC) assigns a lifetime to each content when it is cached [41]. The lifetime is computed depending on node location and content popularity and once the lifetime expires, the content is removed. In contrast to on-path strategies mentioned in the literature, the proposed scheme gathers the caching nodes close to the delivery path as steers, and is referred to as a "near-path" cluster-based scheme.

In summary, most conventional works are characterised by a subset of these six design drivers. The proposed scheme is the first Fog-caching enabled design for IoT 180 scenarios that considers all six drivers of in-network caching. The detailed design of the proposed scheme is explained in next section. 


\section{System Design}

Compared against conventional caching schemes, the proposed Fog-caching scheme offers adjustable, cluster-based, near path and full time caching attributes. The details of these attributes are explained in the following sub-sections.

\subsection{Flexibility: Adjustable Clusters}

The proposed scheme places emphasis on utilising the Fog-nodes for caching purposes. Once the first content request emerges from a group of users, the distributed caching nodes are grouped into $F C$ s. Specifically, based on the topology of all caching nodes within a $F C$, multiple tiers are formed, as seen in Fig. 1. In each cluster, the

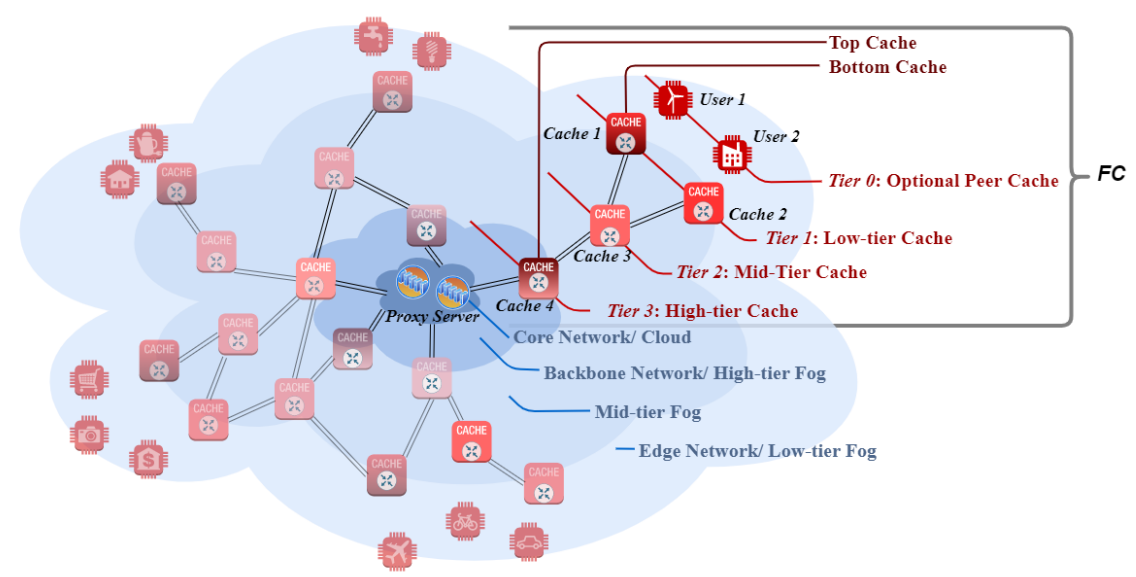

Figure 1: A schematic representation of the proposed $F C$ design scheme with respect to the backbone and Edge, corresponding tiers and individual components Top and Bottom Cache nodes, which are used in the full-time caching attribute.

190

caching nodes close to the end-user devices and the core network are defined as lower and upper tier caches, respectively.

In contrast to ubiquitous caching, the proposed scheme disregards caching capacity of nodes located beyond a preset distance away from the end-user devices. To this end, 195 the highest tier value is assigned in advance, and is determined according to the graph radius of a given network topology. 
After a content request is raised, the lowest-tier Fog-nodes are firstly queried, before continuing to higher tiers. If the requested content exists in the $t^{t h}$ tier nodes, it is delivered to the user and the content is subsequently shifted one tier closer $(t-1)$ to the end-users, ensuring close proximity to users for future content requests. If the requested content is not found in the highest tier, the request message is directly routed to the original content provider at the core network, without any further attempts at the backbone network as happening in ubiquitous caching schemes. In this case, the content is cached into a randomly selected caching node in the highest possible caching tier, which is referred as "Top Cache".

Despite the available storage capacity in end-user devices, sharing it for caching purposes with the rest of the Fog-nodes is optional, rather than enforced. An advantage of the proposed adjustable scheme is the ad-hoc creation of a tier 0 , only when such end-devices are willing to allow their storage to be used as a caching resource. Let us assume that a content request emerges from an IoT end-device, e.g. User 1 in Fig. 1. The corresponding FC spans tier 1 (Cache 1, Cache 2), tier 2 (Cache 3) and tier 3 (Cache 4). If there is at least one end-user device allowing access to it's storage capacity, then tier 0 for this $F C$ is automatically enabled, consisting of all peer caching devices that allow storage access. Note, that peer caching device refers to the shared caching resources of end-user devices as described in [42]. On the other hand, if there is no available end-user device detected, the lowest caching tier would default to tier 1 , consisting of the caching nodes closest to the end-users (Cache 1, Cache 2). Similarly, if there is a content delivery proxy server available at the edge of the network core (Proxy Server in tier 4), the FC can expand towards the core network side to include a higher tier caching location.

\subsection{Near-Path: Caching near the delivery path}

Regarding content placement, most of existing in-network caching schemes adopt an on-path caching scheme because searching for content beyond the delivery path requires additional traffic, thus increasing network load, without guaranteeing success. However, in the IoT Fog environment, the delivery path is unpredictable due to its ad-hoc and continuously evolving nature. Thus, if only caching nodes on the delivery 
path have opportunities to be hit, the nearby (i.e. near-path) resources are not utilised.

In order to trade-off the cost between searching for content and excessive storage, the proposed scheme adopts a hybrid, near-path solution. When the first content request emerges from a new user group, the respective $F C$ is constructed to offer caching services near the delivery path. In this way, the redundancy of contents may be reduced by sharing popular contents among multiple end-user devices. In addition to $F C$, end-user devices, which are less than two hops away from each other (the optional intermediate hop would be either cellular base stations or wifi access points) compose user-clusters $(U C \mathrm{~s})$. The group of users from one or more $U C$ s can share the caching resources in the same near-path $F C$.

\subsection{Full-Time: Combination of Reactive and Proactive Caching}

To reduce the link load during peak time, the full-time caching timing design diverts the arising content allocation traffic to off-peak content delivery hours. Specifically, in the full-time caching proposal, only a single cache of requested content is placed in the allocated $F C$ during the content delivery process, which is the reactive caching slot. Once the user request is successfully satisfied, the cached content would be duplicated and distributed into other caching nodes in the $F C$, during off-peak hours, which is the proactive caching slot. In this way, delivery delay could be reduced by decreasing content populating traffic during the peak of content demand.

\section{System Model}

In this section, the system model of the proposed Fog-caching scheme is described through the permanent character assignment and temporary role allocation. In order to construct a node's potential during a delivery session, node capabilities, such as caching ability, are identified and recorded as permanent characters, once a new content request is generated from a user device.

The role of each caching node is a logical caching coordinate representing the $F C$, tier number, and identity (ID) of the node. During the process for cache allocation, this information is used to determine in which node the requested content should be cached. 

user group that it may serve.

\subsection{Permanent Character Assignment}

In the proposed system, the Fog-network topology graph is denoted by $G=(V, E)$, where $V$ is the set of vertexes in a topology graph (set of nodes) and $E$ represents the graph edges (i.e. links between nodes). Specifically, each node, $v_{i} \in V$, has a unique id, $i$. Each node $v_{i}$ may belong to one or more of the following character sets:

1. Set of user devices, $U$ : Includes end-user devices that initiate content delivery sessions by sending request messages for contents.

2. Set of forwarding hops, $H$ : Includes nodes with message forwarding capability.

3. Set of content providers, $P$ : Includes nodes that are original content providers. In the case of user generated content, the node is also a user device, thus $v_{i} \in(U \cap P)$.

4. Set of caching hops, $C$. Includes nodes with caching capacity. Such node may either be a standalone caching node, an ICN router, $v_{i} \in(C \cap H)$, or a peer user allowing access to its storage, $v_{i} \in(C \cap U)$.

\begin{tabular}{ll}
\hline Character & Node Set \\
\hline End-user device & $U=\left\{v_{i}\right\}, i \in[10,15]$ \\
Caching hop & $C=U \cup\left\{v_{i}\right\}, i \in[1,6]$ \\
Forwarding hop & $H=\left\{v_{i}\right\}, i \in[1,8]$ \\
Content provider & $P=\left\{v_{9}, v_{10}, v_{13}\right\}$ \\
\hline Caching \& Forwarding hop & $C \cap H=\left\{v_{i}\right\}, i \in[1,6]$ \\
End-user \& Content provider & $U \cap P=\left\{v_{10}, v_{13}\right\}$ \\
\hline
\end{tabular}

Table 1: Character assignment for all nodes in the example topology of Fig. 2 All end-user devices are able to cache content, and end-users $v_{10}, v_{13}$ are also original content providers.

Fig. 2 shows an example topology to illustrate the character allocation. Access points $v_{1}$ and $v_{2}$ are connected with ICN routers $v_{3}-v_{5}$ and $v_{4}-v_{6}$, respectively, at the network Fog. 


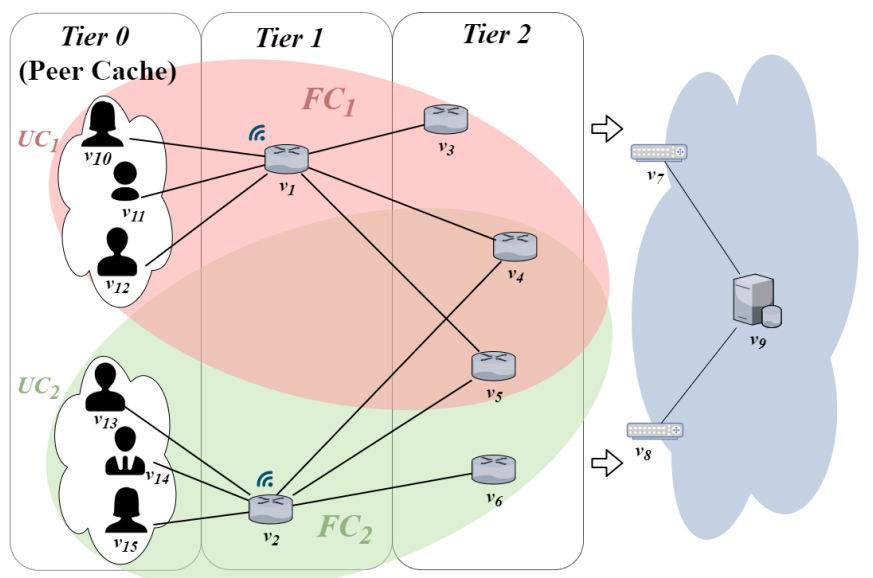

Figure 2: An example of a Fog network topology to illustrate the character and role allocation, based on the capabilities and tier of every node. The diagram illustrates the borders of $U C_{1}, U C_{2}, F C_{1}$ and $F C_{2}$. Note, that nodes $v_{4}$ and $v_{5}$ belong to both $F C_{1}$ and $F C_{2}$.

Since nodes $v_{1}-v_{6}$ have both caching and forwarding capability, they belong to both $\mathrm{C}$ and $\mathrm{H}$ sets. Nodes $v_{7}-v_{9}$ are at the edge of the core network and are only forwarding devices without caching capacity. Node $v_{9}$ at the core network is the source of original content. In addition, since this paper is focusing on user generated video, two end-user devices are assumed to be potential content providers $\left(v_{10}, v_{13} \in P\right)$. The character assignment for all nodes in Fig. 2 is summarised in Table 1.

\subsection{Temporary Role Allocation}

As mentioned in the design section, there are two types of clusters in the proposed caching scheme, which are $U C$ and $F C$. The nodes within the same $F C$ work collaboratively to concurrently serve one or more $U C$ s and each end-user device may belong to at least one $U C$.

Fig. 2 shows users $v_{10^{-}} v_{12}$ clustered in $U C_{1}$, while users $v_{13}-v_{15}$ are clustered in $U C_{2}$. If a user devices does not have caching capacity then it can only be included in a $U C$, but not in a $F C$. Note, that it is possible for a Fog node to belong to more than one $F C$ s, such as $v_{4}$ and $v_{5}$ that belong to both $F C_{1}$ and $F C_{2}$. 


\begin{tabular}{c|llll|lll}
\hline \multirow{2}{*}{ Node } & \multicolumn{2}{|c}{ If no end-users can cache } & & \multicolumn{3}{c}{ If end-users can cache } \\
\cline { 2 - 3 } \cline { 5 - 7 } & Cluster & tier & Role & & Cluster & tier & Role \\
\hline$v_{2}$ & $F C_{2}$ & 1 & $(2,1,2)$ & & $F C_{1}$ & 1 & $(2,1,2)$ \\
$v_{4}$ & $F C_{1}$ & 2 & $(1,2,4)$ & & $F C_{1}$ & 2 & $(1,2,4)$ \\
& $F C_{2}$ & 2 & $(2,2,4)$ & & $F C_{2}$ & 2 & $(2,2,4)$ \\
$v_{5}$ & $F C_{1}$ & 2 & $(1,2,5)$ & & $F C_{1}$ & 2 & $(1,2,5)$ \\
& $F C_{2}$ & 2 & $(2,2,5)$ & & $F C_{2}$ & 2 & $(2,2,5)$ \\
$v_{11}$ & N/A & N/A & N/A & & $F C_{1}$ & 0 & $(1,0,11)$ \\
$v_{14}$ & N/A & N/A & N/A & & $F C_{2}$ & 0 & $(2,0,14)$ \\
\hline
\end{tabular}

Table 2: Logical caching coordinates (roles) of five nodes from the example Fog topology of Fig. 2 in two scenarios: When no end-user has caching capability and when end-users do have caching capacity.

A node's role encodes its position in the Fog network topology. For example, in Fig. 2. caching node $v_{3}$ has role $(1,2,3)$, represent $F C_{1}$, tier 2 and node $i d=3$. Table 2902 presents several other node roles from Fig. 2 in the case when all end-users are able to cache and when they can not.

\section{System Implementation}

The system implementation of the proposed scheme consists of three steps: 1) Preparation, 2) Request message routing and delivery and 3) Content delivery to enduser and caching content closer to end-users for future use. There are 8 algorithms presented in this section. Algorithms 1-3 are used during the first step, algorithms 4-6 during the second step and the final 2 algorithms during the third step.

\subsection{Step 1 Preparation}

The preparation step, Alg. 11 is responsible for constructing the $F C$ for a specific user group based on the individual node character assignment and role allocation, as described in Section 4 A Boolean logic peer flag, $F_{p}$, is used to indicate whether tier 0 exists. Only when there are peer end-user devices with caching character in the same tier as the content requester, is $F_{p}$ set to 1 . In addition, during the preparation 


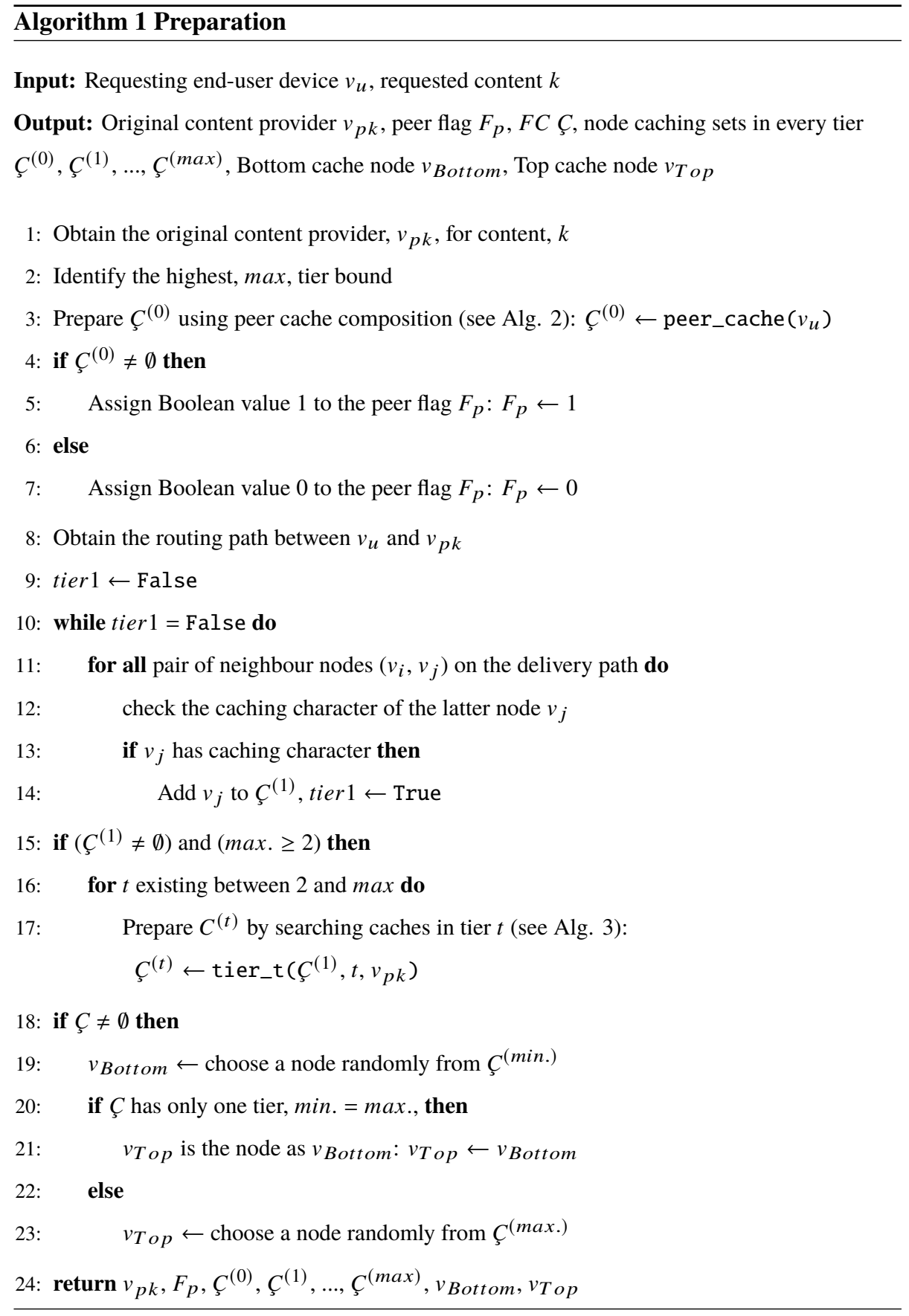


step a Bottom and Top caching nodes are nominated to be used during the request message routing (step 2) and caching (step 3), respectively, as will be detailed in later subsections.

More specifically, the original content $k$ provider node, $v_{p k}$, where $p k$ is the node's id, is found and recorded (line 1) along with identifying the highest, $\max$, tier number (line 2). Then, by calling function peer_cache() (Alg. 2), the caching character of every peer user-device is detected. If there are peer devices with caching character, they are included in tier 0 set, $C^{(0)}$, and the peer flag, $F_{p}$ is set to 1 . Otherwise, tier 0 is empty and $F_{p}$ is set to 0 (lines 3-7).

Once the routing path from the requesting end-user, $v_{u}$, to the original content provider $v_{p k}$ is identified (line 8), the node for tier 1 is found along the delivery path ${ }_{315}$ and put in set $C^{(1)}$ (lines 9-14) and, then nodes belonging in tier $t$ are included in set $C^{(t)}$ (lines 15-17). Finally, for future routing and caching purposes, the Bottom, $v_{\text {Bottom }}$, and Top, $v_{T o p}$, cache nodes are randomly selected from the lowest and highest tiers of $C$, respectively (lines 18-23). Note, that in the case when there is only one tier, the Bottom and Top cache nodes are the same node (line 21).

The peer cache composition function peer_cache(), shown in Alg. 2, aims to collect all peer nodes with caching character in set $C^{(0)}$. Initially, the set of peer caching nodes $C^{(0)}$ is an empty set (line 1). Then, for all end-user devices in $U C$, besides $v_{u}$ itself, their caching character and hop distance from the requesting end-user, $v_{u}$, are checked. If an end-user's distance is equal to or less than two hops away from $v_{u}$ (e.g. users $v_{10}$ and $v_{11}$ in Fig. 2 are two hops $\left(v_{1}\right)$ away) and has caching character, then it is included in tier 0 set $C^{(0)}$ (lines 3-4).

Similarly, the tier cache composition function tier_t(), shown in Alg. 3, aims to collect all tier nodes higher than tier $1, t \geq 2$, in set $C^{(t)}$. Initially, the set of tier caching nodes $C^{(t)}$ is an empty set (line 1$)$. Any node, $v_{j}$, with caching character and $(t-1)$ 3зо hop distance from tier 1 will be included in the set $C^{(t)}$ (lines 3, 4). For example, in Fig. 2, node $v_{3}$ from $v_{1}$ is 1 hop away. In general, nodes in tier $2, t=2$, will be $t-1=1$ hops away from tier 1 nodes, nodes in tier 3 will be $t-1=2$ hops away from tier 1 nodes, an so forth. 

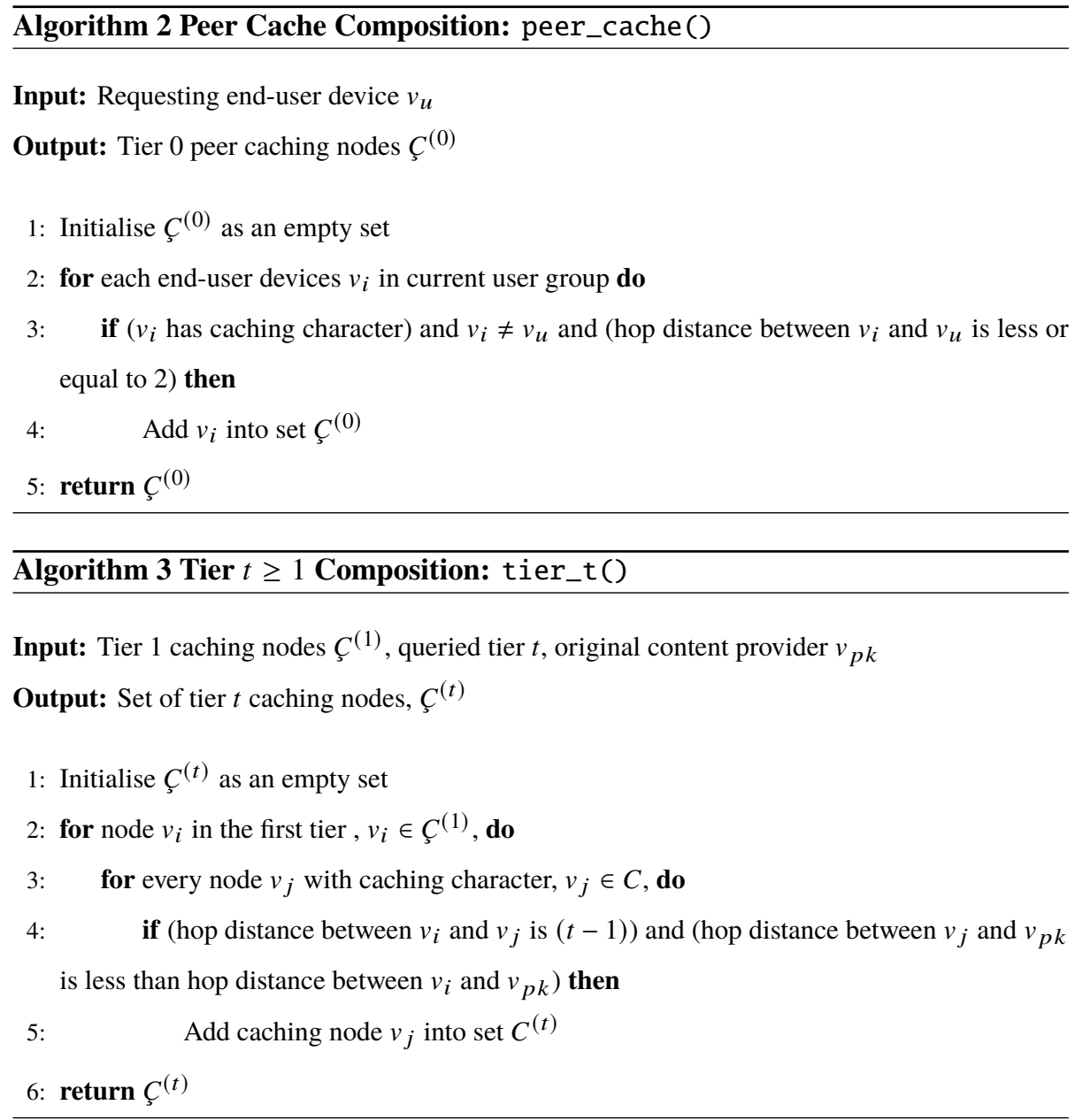

\subsection{Step 2 Request Routing and Delivery}

335 During the request message routing and delivery, the Bottom cache node, selected in the preparation step, is delegated to forward the content request message in order to identify in which node and tier the requested content is located. To this end, tier 0 is initially searched (Alg. 4), since it is the closest to the end-user. If the content is not found in tier 0 and if there are higher tiers, searching continues in those higher tiers ${ }_{340}$ (Alg. 6), i.e. in in-network nodes. If there are no available caching nodes in higher tiers, the content request message is forwarded directly to the original content provider (Alg. 5). 


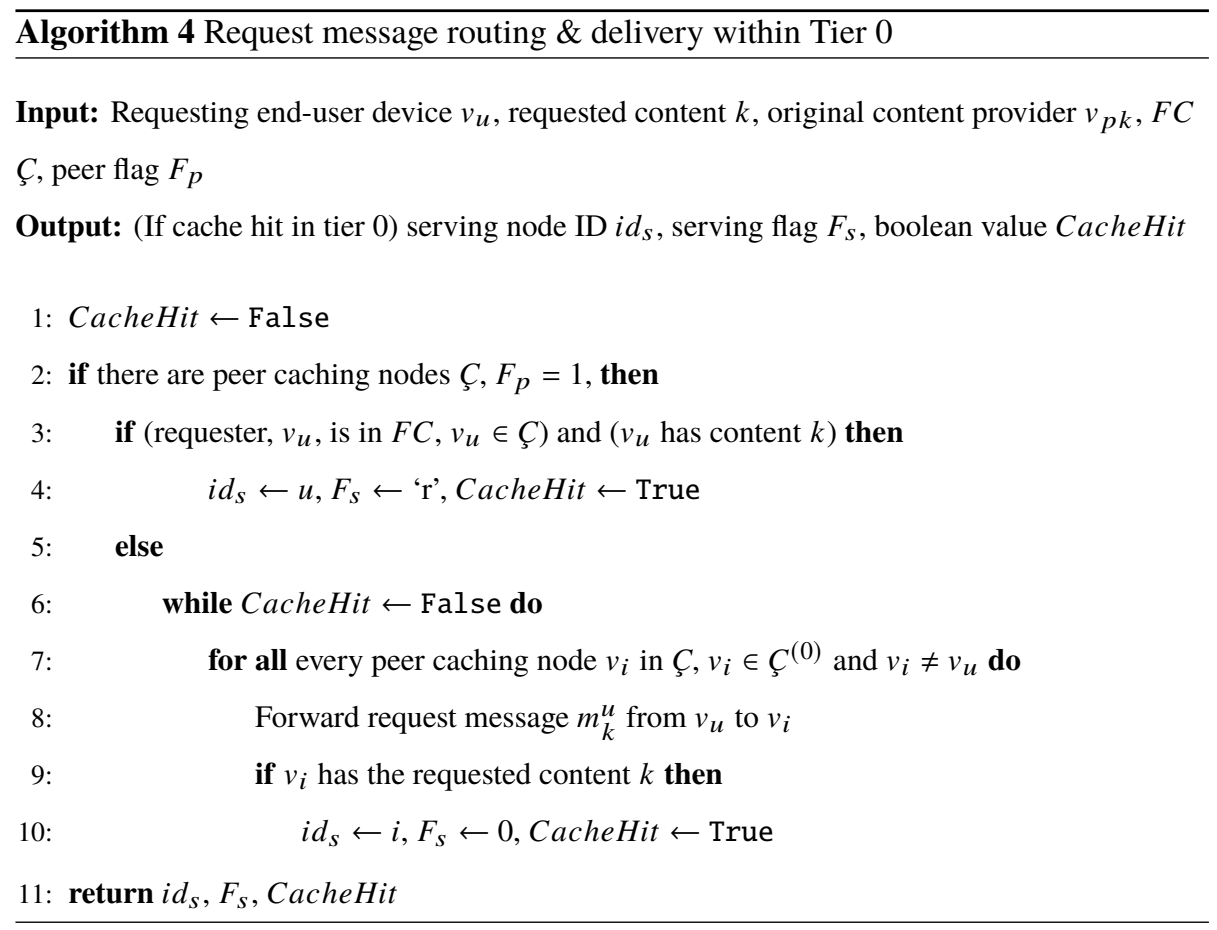

Two important variables, the serving flag and the serving node id, are introduced in algorithms 4-6. The serving flag, $F_{s}$, is used to record the tier number, where the content is ultimately found. It can either take an integer value from $\{0 \ldots$ max $\}$ to indicate the tier $t$ value, or the value 's' (source), if the content is found at the original source. Lastly, if the content is located at the requesting node itself, in case the content was previously cached there, the $F_{S}$ flag will take the value 'r' (requester). In addition, once the content is found in a caching node, the serving node id variable, $i d_{s}$, simply stores the id number of the node that holds and serves the content. This information will be later used in the content caching process.

Alg. 4 searches for the requested content both within the requesting node itself, $v_{u}$, and all of its peer nodes, $v_{i}$. Initially, the requesting node checks whether itself holds the content. If it does, then the $i d_{s}$ value is assigned with the ID $u$ of user $v_{u}$ and the serving flag $F_{s}$ is assigned to ' $r$ ' to represent a local cache hit (lines 3-4). Otherwise, if the cache of requested content $k$ cannot be found locally, the request is forwarded to all the other nodes, $v_{i}$, in $C^{(0)}$, until a cache hit occurs (lines 7-8). When a cache hit 


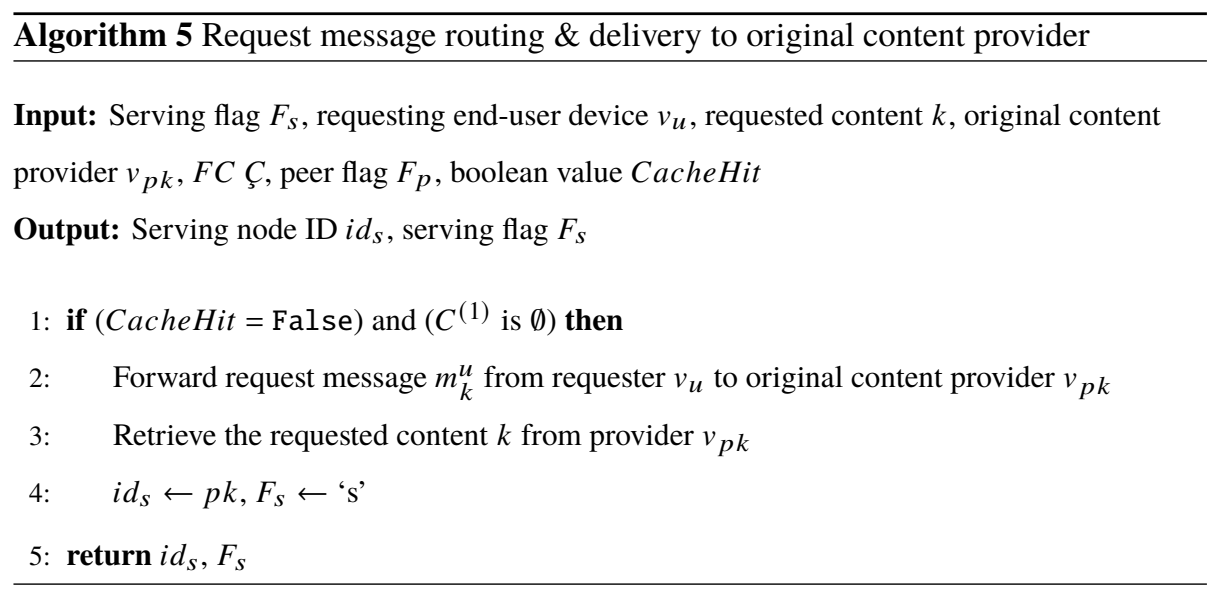

occurs, $i d_{s}$ is assigned the id of the node $v_{i}$ and $F_{s}$ is set to ' 0 ' to indicate a zero-tier peer node cache (line 9).

If a cache hit has not occurred within tier 0 (therefore the serving flag is not set) and there are no caching nodes in higher tiers, i.e. $C^{(1)}=\emptyset$, the request message is forwarded directly from user $v_{u}$ to the original content source $v_{p k}$, as shown in Alg. 5 (lines 1-2). Once the content is received from the content provider, the serving flag $F_{s}$ and the $i d_{s}$ are assigned with 's' and the ID of the content provider, $p k$, respectively (lines 3-4).

Alternatively, if a serving cache node has not been found yet, CacheHit $=$ False, and there are caching nodes in higher tiers, $C^{(1)} \neq \emptyset$, the request message $m_{k}^{u}$ would be traversed from the lowest to the highest tiers in search of a cache hit, as shown in Alg. 6 If $F_{p}$ is 1 , it means there is peer cache in $C$ and, therefore the Bottom cache node ${ }_{370}$ exists in tier 0 . In this case, the $v_{\text {Bottom }}$ node has already obtained the request message during the peer searching process (Alg. 4 ) and is not part of Alg. 6

Otherwise, if $F_{p}$ is 0 (line 2), message $m_{k}^{u}$ is forwarded from $v_{u}$ to the Bottom cache node $v_{\text {Bottom. }}$. In the first tier traversing, the request message $m_{k}^{u}$ is broadcasted from $v_{\text {Bottom }}$ to all the nodes in the first tier nodes in sequence (lines 5-11). If a cache 375 hit occurs, the $i d_{s}$ would record the provider ID and $F_{S}$ is set to 1 , representing that a cache hit occurred in the first tier. In this case, the request routing and delivery process is finished and the content delivery and caching process in step 3 would be triggered. 
Nevertheless, if there is no cache hit in the first tier, the last traversed node in the first tier is set as a temporary gate, $v_{g}$, tasked to broadcast the request message to the next tier (lines 13). Similarly, if there is no cache hit, the request message is recursively delivered to each higher tier (lines 15-23). Once the highest, $\max$, tier is reached and, still, there is no cache hit, then the request is routed from $v_{u p}$ to the original content provider $v_{p k}$ (lines 24-27). 


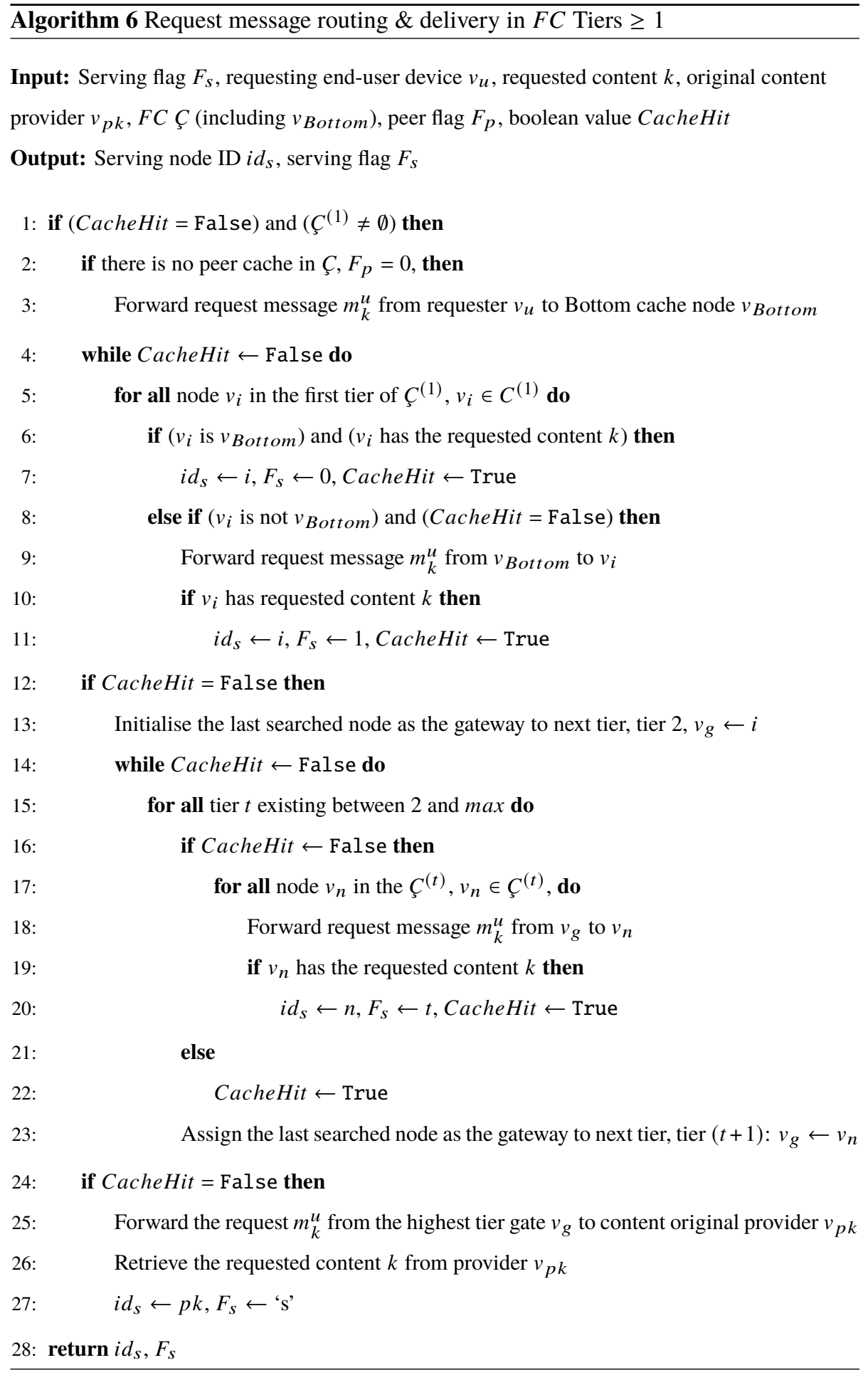




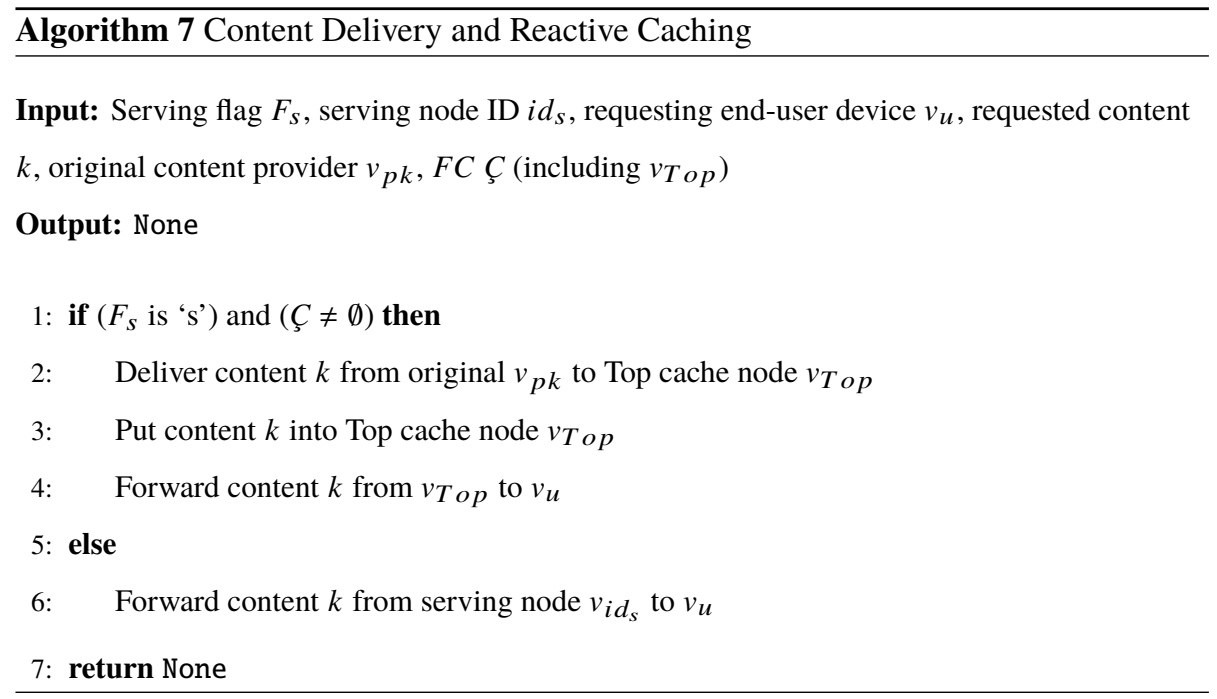

\subsection{Step 3 Content Delivery and Caching}

Once the requested content is found in any of the above cases (tier 0 , tier $\geq 1$, or original source), the content is delivered back to the requesting user whilst a cache of the content is stored within the $F C$, following either a reactive and/or a proactive caching scheme. This step is powered by the full-time caching attribute of the proposed scheme.

Once the requested content $k$ is delivered back to user $v_{u}$, a reactive caching strategy would leave a single cache $k$ in the $F C$. The proactive caching process will then populate the $F C$ with additional content caches. Specifically, as shown in Alg. 7, only when the content is found in the original content provider $\left(F_{S}=\right.$ 's') and there are nodes in the Fog-Cluster $(C \neq \neq \emptyset)$ (line 1$)$, is reactive caching activated to leave a cache in $v_{T o p}$ (lines 2-4). In this case, a duplicate of content $k$ is firstly forwarded to the Top cache node, $v_{u p}$ (line 2). Once the content is cached in $v_{u p}$, it is forwarded back to user $v_{u}$ (lines 3-4).

Regarding the proactive caching operation in Alg. 8, if there is a caching node existing in the $F C$ and the content request has been satisfied by the original content provider $\left(F_{s}=\right.$ 's'), a cache should already exist in the Top cache node, from the reactive caching process. In this case, the content is redistributed from $v_{T o p}$ to all the caching 
nodes in the highest tier caches (lines 2-5). If the highest tier is the end-user tier, an additional content is cached in the user device $v_{u}$, if it has caching character (lines 6-7).

If the $v_{i d_{s}}$ is the caching node in any tier $t \geq 2$, the requested content is moved to caches located at one tier lower $\left(F_{S}-1\right)$. In this case, the contents in tier $F_{s}$ are removed to reduce the caching redundancy (lines 8-13). In addition, if the receiver device has caching character, another content is leaved there as well (lines 14-15) In another case, if there is peer cache existing in $C$ and $v_{i d_{s}}$ is one of peer caches $\left(F_{s}=0\right)$, the content should be forwarded from the serving peer cache to user $v_{u}$ (line 18-19).

\section{Experimental Set Up}

In order to evaluate the proposed in-network caching scheme without binding to any specific ICN architecture, the caching simulator Icarus [11] is chosen to conduct the experiments, running on an Intel Core i5-4200M at 2.5Ghz, 4 core CPU with 4GB of RAM.

In this section, a suitably designed topology for Fog caching evaluation purposes is provided. The parameter configuration, topology design and performance metrics are elaborated in the following subsections.

\subsection{Simulation Parameters}

In our simulation, the parameters could be categorized into three groups, which are ${ }_{420}$ request message parameters, content parameters and caching parameters. The detailed configuration in these three categories are successively explained as follows.

The request message parameters involve the arrival distribution, generation rate and Icarus' warm up and measured messages. The warm up messages are traffic generated in the initial phase to populate the caching nodes with cached contents. Whereas the measured messages are the actual request messages collected for evaluation purposes once the warm up phase finishes.

The request message arrival is following the Poisson distribution, which is the most widely used distribution in conventional caching experiments [43, 44]. The content generation rate is set to 12 request messages per second, since this is Icarus' default 


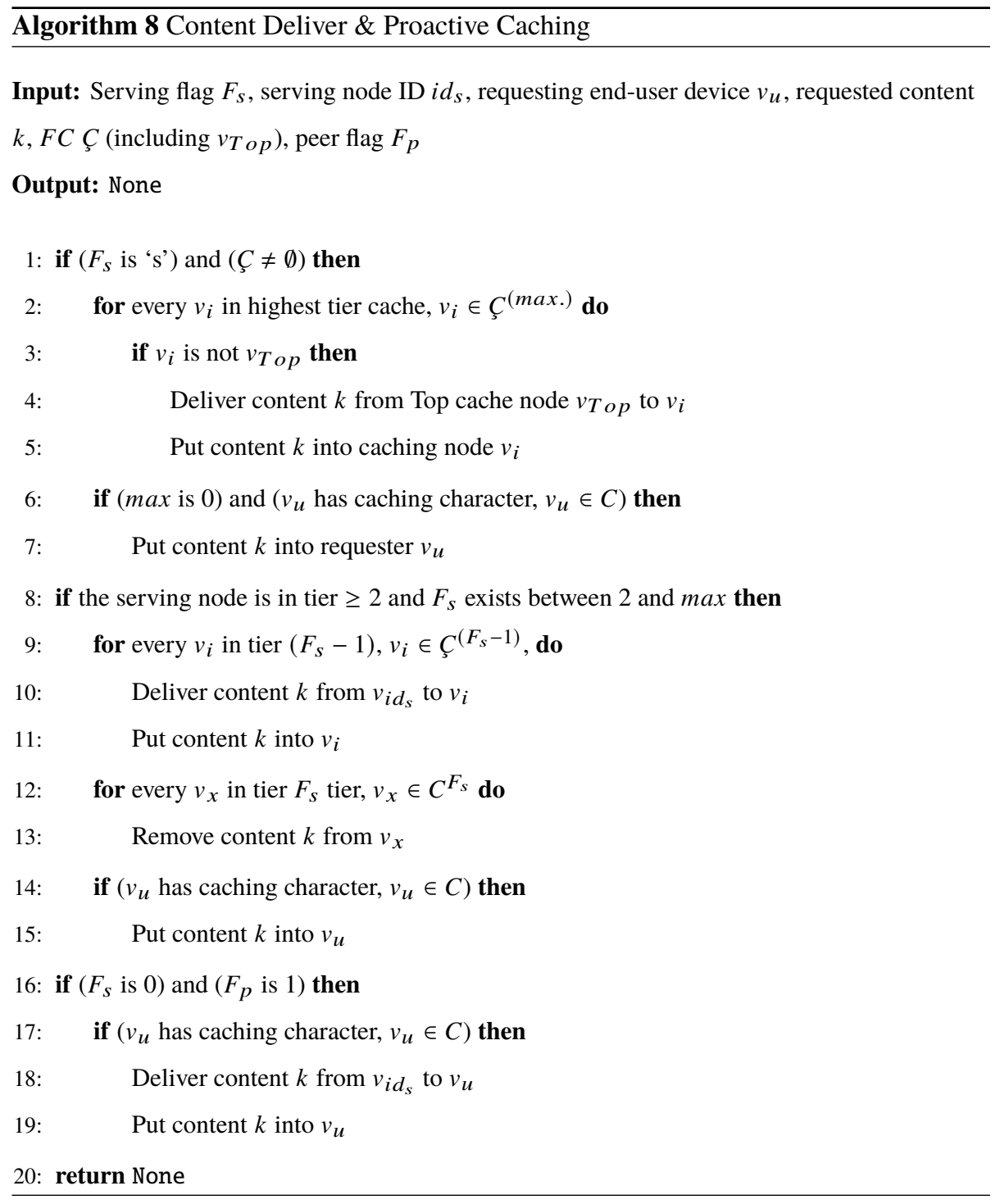


setting and does not negatively impact the performance of the host machine running the simulations. In addition, the other two request parameters, i.e. the number of warm up messages and measured messages, are configured as $3 \times 10^{5}$ and $6 \times 10^{5}$ messages, respectively.

Content parameters consist of number of contents, $|K|$, and content popularity parameter, $\alpha$. The number of contents varies considerably depending on the simulated scenario. For example, the number of contents pertaining to user generated videos would be much larger than in video-on-demand scenarios. In our experiments, the number of contents is $3 \times 10^{5}$ individual contents. Zipf's law, $\frac{1}{|K|^{\alpha}}$, conventionally employed in related work, is chosen for the content popularity distribution. The $\alpha$ parameter in Zipf's law reflects the concentration of user preference and ranges from 0.75 to 1.25 in the experiments. The greater the $\alpha$ value is, the more concentrated the user preference is. In other words, assuming $\alpha=1.25$, more request messages are asking for the same content than if $\alpha=0.75$.

For the third parameter category, caching parameters include network caching capacity, storage capacity allocation and content eviction algorithm. For fairness purposes, the sum of storage capacity of caching nodes in different topologies should be consistent. Therefore, network caching capacity $(C C)$ is set as a constant value for all topology scenarios. This constant value denotes the ratio of total network caching capacity over number of contents, $|K|$, as in Eq. 1 .

$$
C C=\frac{1}{|K|} \sum_{c_{i} \in C}\left|c_{i}\right|,
$$

where $c_{i}$ is the caching capacity of node $i$.

In this work, the network caching capacity ranges from $0.2 \%$ to $5 \%$. Under the uniform capacity allocation scheme, the storage capacity of all caching nodes is equal. Therefore, the caching capacity of each caching node is the ratio of $C C$ over the number of caching nodes. Regarding the used content eviction algorithm, the proposed scheme implements the LRU, which has a constant time complexity, but also keeps 450 the consistency with most of existing ubiquitous caching schemes. Based on the above request, content and caching parameters, each analysed caching scheme is analysed with 10 possible values for network caching capacity and 11 different $\alpha$ values for content 


\begin{tabular}{ll}
\hline Parameters & Configuration \\
\hline Request arrival & Poisson's Distribution \\
Request rate & 12 requests per second \\
Num. of warm up request & $3 \times 10^{5}$ messages \\
Num. of measured request & $6 \times 10^{5}$ messages \\
Num. of contents & $3 \times 10^{5}$ contents \\
Content popularity & Zipf's law, $\alpha=0.75,0.8, \ldots, 1.25$ \\
Network caching capacity (CC) & $0.2 \%, 0.6 \%, 1 \%, 1.4 \%, 1.8 \%$, \\
& $2.2 \%, 2.6 \%, 3 \%, 4 \%, 5 \%$ \\
Storage capacity allocation & Uniform \\
Content eviction algorithm & Least Recently Used (LRU) \\
Num. of iterations for each experiment & 3 \\
Performance metrics & Latency, Cache Hit Ratio, \\
& Internal Link Load, Path Stretch \\
\hline
\end{tabular}

Table 3: Experimental set-up parameters and configuration.

popularity, as shown in Table 3 thus, composing 110 scenario configurations in total. Each evaluated scenario is iterated three times and the average value for each metric is calculated and used for comparison purposes against benchmark caching schemes. The simulation configuration is summarised in Table 3 .

\subsection{Topology Configuration}

Although many research works are focusing on Edge or Fog caching, there is lack of appropriate topology consideration for Fog caching evaluation purposes. The conducted experiments aim to address a lack in related work by simulating IoT scenarios at network Fog, which exhibiting request traffic for both user generated and conventional, ondemand content. In addition, the evaluated topologies in other related works are either small scale and coupled to specific network technologies [45, 46] or large scale based on an existing backbone topology [47, 48] that is not appropriately structured to simulate a

${ }_{465}$ Fog environment. To this end, a novel simulation topology is proposed, which includes user devices that not only send requests, but also participate as caching nodes and may 
even provide user generated content. In addition, ICN routers, traditional forwarding nodes, and content providers in core network are also included in the topology design.

Specifically, the design of our topology is based on a traditional tree structure as shown in Fig. 2 The root node, $v_{9}$, represents the core network, where there is an original content source located. The children of the root are the cloud-edge nodes, $v_{7}$ and $v_{8}$, which are connected to the Fog's outer edge, i.e. the highest caching tier in $F C$. Since the hops between the Fog's outer edge and cloud-edge do not take responsibility in the proposed Fog-based scheme, they are not utilised in our topology implementation.

475 At the Fog, ICN nodes $v_{3}-v_{6}$ are connected with access points $v_{1}$ and $v_{2}$ at network edge, each of them have both caching and forwarding characters. For $v_{1}$ and $v_{2}$, they are connected to several user-devices with allocated end-user characters, as described in Section 4.1

In our experimental topology, there are six end user devices and all of them have ${ }_{480}$ caching character. Since there are user generated contents in IoT environment, two of the available user devices are also assigned with content source character. Besides, to place more emphasis on the network fog, the potential bottleneck at the core network is eliminated by assigning unlimited bandwidth for those links during the simulations. Thus, any delay would be caused by the Fog node links, rather than the backbone.

\subsection{Performance Metrics}

There are four metrics used for performance evaluation, which are latency $(L A T)$, cache hit ratio $(C H R)$, internal link load $\left(L L_{i n}\right)$ and path stretch $(P S)$. The definitions of these metrics are described as follows, [11]:

Latency: Mean latency is the average delay from each request $m_{k}^{u}$ generated until the content $k$ is delivered to the user $v_{u}$. The amount of measured requests are denoted by the size of request set $|M|$, generated during the simulation time $T$. In order to calculate the delivery delay of request $m_{k}^{u}, D E L(m)$, the forwarding operations of both request message, $m$, and content objects, $o$, for request $m_{k}^{u}$ are collected in sets $F(m)$ and $F(o)$, 
respectively. Then, the deliver delay of message $m$ is given by Eq. 2 ;

$$
D E L(m)=\sum_{\left(v_{a}, v_{b}\right) \in F(m)} d\left(v_{a}, v_{b}\right)+\sum_{\left(v_{a}, v_{b}\right) \in F(o)} d\left(v_{a}, v_{b}\right),
$$

495

where the link delay between node $v_{a}$ and $v_{b}$ is denoted as $d\left(v_{a}, v_{b}\right)$.

Finally, the latency $L A T$ metric is defined as the average delivery delay of message $m$, as in Eq. 3

$$
L A T=\frac{1}{|M|} \times \sum_{m \in M} D E L(m)
$$

Cache Hit Ratio: The cache hit ratio metric is a portion of the requests satisfied by caching nodes, rather than servers. Let us assume that there are $M$ request messages being served in total and the session providers are either content sources or Fog-caches. If a cache of requested content $k$ can be found in one of the Fog nodes in $F C$, a cache hit is added in counter $H_{c}$. On the other hand, if $m$ can not be satisfied within the $F C$, it will be served by the original content source, and this is counted as a server hit $H_{s}$. Therefore, the cache hit ratio is defined in Eq. 4

$$
C H R=\frac{H_{c}}{H_{s}+H_{c}}=\frac{H_{c}}{|M|}
$$

Internal Link Load: The internal link load $\left(L L_{i n}\right)$ is the average traffic load of links at the network Fog. Let $m$ be the message size of each request and $o_{k}$ be the content size of requested object $k$. In Icarus, the content size are uniform, so the size of content object can uniformly be denoted as $o$. The amount of contents and requests passed by link $e$ are represented by $N_{o}(e)$ and $N_{m}(e)$, respectively. The link load of link $e$ is denoted as $L L_{e}$, which is defined as Eq. 5 .

$$
L L_{e}=\frac{1}{T}\left[|m| \times N_{m}(e)+|o| \times N_{o}(e)\right]
$$

Moreover, let $E_{\text {in }}$ be the set of internal links in the Fog topology. The mean internal link load for the used links $L L_{i n}$ in the Fog topology is calculated as shown in Eq. 6

$$
L L_{i n}=\frac{1}{\left|E_{i n}\right|} \sum_{e \in E_{i n}} L L_{e}
$$


Path Stretch: Path stretch $(P S)$ is the metric reflecting the length of delivery path. In the ubiquitous caching scenario, the closer to the requesting node the cache hit happens, the lower the $P S$ value is. Specifically, $P S$ is the ratio of the total length of delivery path over the length of shortest routing path between user $v_{u}$ and $v_{p k}$. The length of shortest path between nodes $a$ and $b$ is denoted as $\operatorname{SP}(a, b)$. The path stretch of request message $P S_{m}$ and content object $P S_{o}$ is defined in Eq. 7 and 8 , respectively. The $P S$ uses the average path stretch value of request messages and content objects.

$$
\begin{gathered}
P S_{m}=\frac{1}{|M| \times\left|S P\left(v_{u}, v_{p k}\right)\right|} \sum_{m \in M} \sum_{\left(v_{a}, v_{b}\right) \in F(m)}\left|S P\left(v_{a}, v_{b}\right)\right| \\
P S_{o}=\frac{1}{|M| \times\left|S P\left(v_{p k}, v_{u}\right)\right|} \sum_{m \in M} \sum_{\left(v_{a}, v_{b}\right) \in F(o)}\left|S P\left(v_{a}, v_{b}\right)\right| \\
P S=\frac{P S_{m}+P S_{o}}{2}
\end{gathered}
$$

\section{Results and Discussion}

For comparison evaluation, eight caching schemes are chosen as benchmarks, which are Edge Caching, Consumer Caching (CSM), LCE, LCD, RAND, CL4M, Random Bernoulli and ProbCache. In particular, Edge Caching and CSM are popular schemes focusing on caching node placement. Edge Caching caches the content in distributed caching hops located at the network edge, e.g. proxy servers for content delivery network (CDN). CSM caches popular contents locally in end-user devices. LCE and LCD are two classic content placement strategies for ubiquitous ICN caching. The former one cache the requested content to all the caching hops on the delivery path, while the latter one moved the cached content towards the end-users hop-by-hop along the delivery path when the content is repeatedly requested. In random-based content placement, RAND caches content in a randomly selected caching node on the delivery path, while Random Bernoulli randomly inserts content in an on-path node with probability $p$ [11]. CL4M, 510 a more recent caching scheme is based on graph-based centrality [13]. ProbCache 
calculates the probability of caching specific content according to a node's position and remaining caching space on the delivery path [14].

In the conducted 110 experiments discussed earlier, four of the benchmark schemes, LCE, CL4M, Edge Caching and CSM, present comparable performance for the evaluation metrics of latency, cache hit ratio and path stretch. A sample representation of these results is presented in Table 4 . It is worth noting that for the internal link load metric, CSM performs better than the rest of the schemes. This is justified because the CSM scheme caches content at the user side, thus, resulting in the lowest link load results, as seen in Table 4 For this reason, CSM is chosen as the representative benchmark among these four schemes. However, the remaining four schemes, LCD, RAND, Random Bernoulli and ProbeCache, vary significantly and are preserved in the following comparison results.

\subsection{Cache Hit Ratio}

The cache hit ratio is one of the most important metrics among the four investigated in our evaluation, because a high cache hit ratio implies a reduced workload at the core network and balances the content requests among the available cache resources. Conventionally, a high cache hit ratio implies that the content requests are satisfied by nearby caching nodes, rather than the remote original content source, thus incurring a low latency along with low link load and short path stretch. The proposed scheme performs consistently better in comparison to the five benchmarks in all the conducted

\begin{tabular}{ccccc}
\hline Caching Scheme & Latency & Int. Link Load & Cache Hit Ratio & Path Stretch \\
\hline LCE & $83.643(\mathrm{~ms})$ & 1948.545 (bytes) & $43.1 \%$ & $54.2 \%$ \\
CL4M & $84.214(\mathrm{~ms})$ & 1947.037 (bytes) & $42.7 \%$ & $54.8 \%$ \\
Edge Caching & $84.264(\mathrm{~ms})$ & 1945.853 (bytes) & $42.7 \%$ & $54.4 \%$ \\
CSM & $82.605(\mathrm{~ms})$ & $\mathbf{1 4 1 9 . 1 5 8}$ (bytes) & $42.6 \%$ & $45.9 \%$ \\
\hline
\end{tabular}

Table 4: Performance results for LCE, CL4M, Edge Caching and CSM with content popularity skewness $\alpha=1$ and network caching capacity $C C=3 \%$. These four schemes perform similarly in all metrics with the exception of internal link load for CSM. 


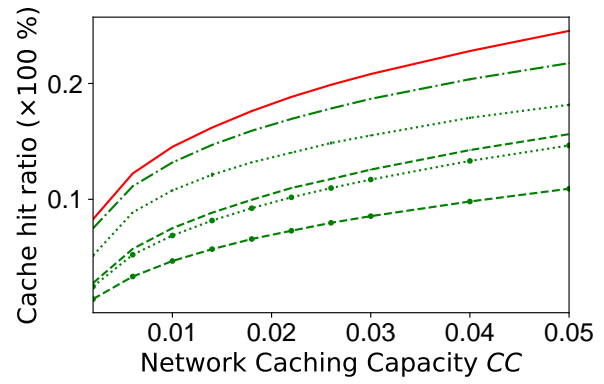

(a) $\alpha=0.75$

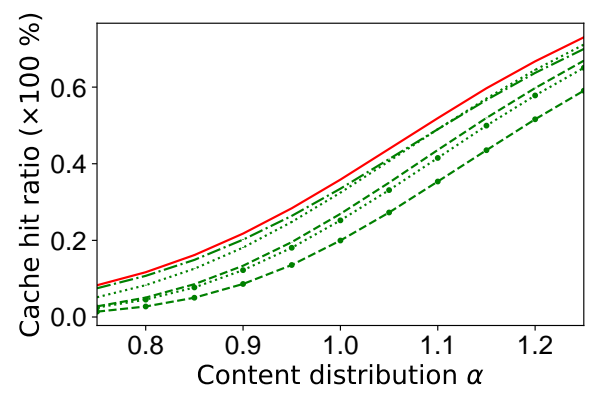

(c) $C C=0.2 \%$

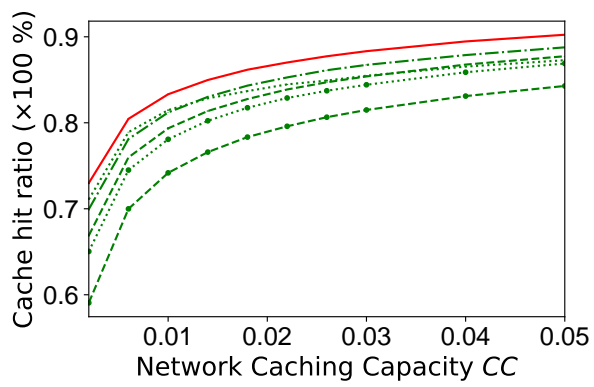

(b) $\alpha=1.25$

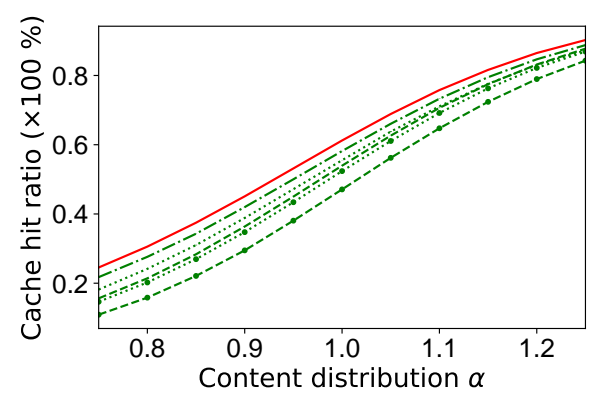

(d) $C C=5 \%$

- The Proposed Scheme

-.-. LCD

---- Random Bernoulli

Figure 3: Cache Hit Ratio of proposed scheme against benchmark schemes. 
experiments where the skewness and $C C$ parameters vary across all examined values, as shown in Table 3. Fig. 3a and Fig. 3b show the cache hit ratio performance of all analysed schemes with varying caching capacity $(C C)$ and fixed content popularity skewness, $\alpha$, equal to 0.75 and 1.25, respectively. On the other hand, Fig. 3c and Fig. ${ }_{535}^{3 \mathrm{~d}}$ plot the cache hit ratio performance for all six caching schemes under varying $\alpha$ values, but with $C C$ equal to $0.2 \%$ and $5 \%$, respectively.

Generally, in all the evaluated scenarios, the average cache hit ratio of the proposed scheme is $51.8 \%$, which is $4.9 \%$ higher than the second best scheme, i.e. LCD (with an average cache hit ratio of 49.4\%). As shown in Fig. 3a, the cache hit ratio of the proposed scheme improves by up to $2.7 \%$ in comparison to LCD. In Fig. 3b, where $\alpha$ $=1.25$, the cache hit ratio has a slight improvement ranging between $1.4 \%$ and $2.8 \%$, in comparison to LCD.

The adjustable $F C$ attribute of the proposed scheme, allows the possibility for enduser devices to act as content caching nodes and serve their neighbours while being in a close proximity. This is one attribute that justifies the better performance of our scheme. However, as it is observed from the Consumer Caching scheme, which is solely based on end-user caching, its performance is less than the proposed scheme under the considered topology. The reason why the proposed scheme succeeds in achieving a higher cache hit ratio performance is because in addition to utilising end-user devices, 550 it also adopts the near-path caching that leverages available caching resources close to the delivery path. It is also worth mentioning that when the content preference becomes less concentrated, i.e. users request a wider variety of content (see Fig. 3a), then there is an increasing impact to the performance of the proposed scheme with increasing $C C$, in comparison to when the content preference is more concentrated (see Fig. 3b).

Figures $3 \mathrm{c}$ and $3 \mathrm{~d}$ show that with increasing $\alpha$ and fixed $C C$ the cache hit ratio performance changes roughly from $10 \%$ to $70 \%$ and from $20 \%$ to $85 \%$, respectively. This performance change is significantly larger than the performance gains achieved when varying the $C C$ values and having fixed $\alpha$ values, shown in Figures $3 \mathrm{a}$ and $3 \mathrm{~b}$. Therefore, the selection process for a caching scheme should mainly be based on the ${ }_{560}$ particular content popularity skewness within the Fog network. 


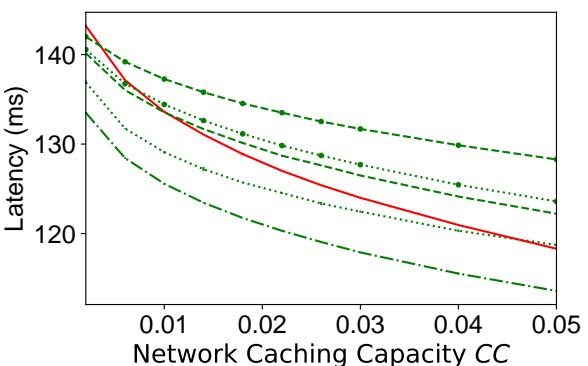

(a) $\alpha=0.75$

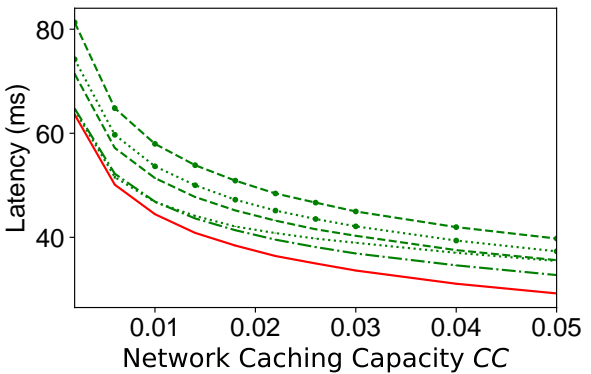

(c) $\alpha=1.15$

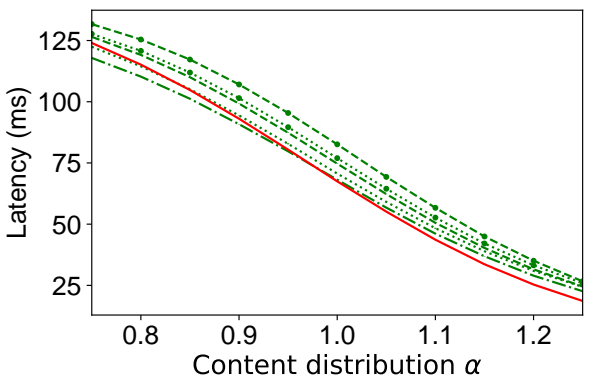

(e) $C C=3 \%$

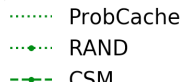

$\rightarrow-$ CSM

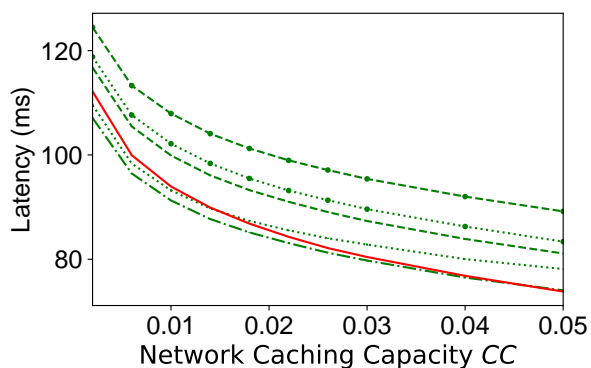

(b) $\alpha=0.95$

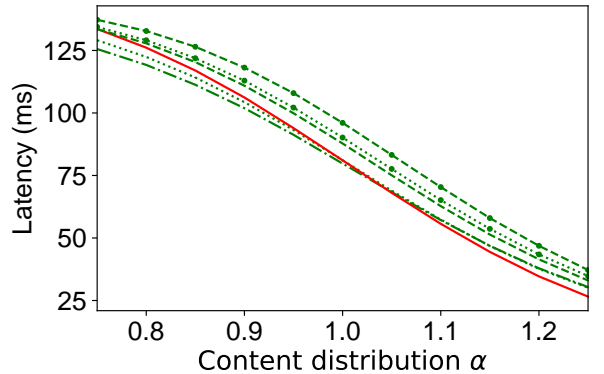

(d) $C C=1 \%$

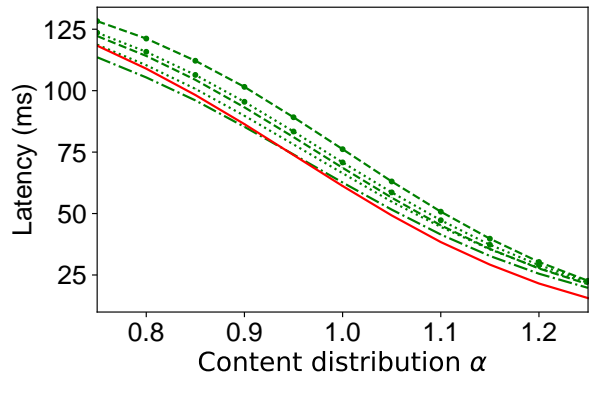

(f) $C C=5 \%$

— The Proposed Scheme

-.-. LCD

---- Random Bernoulli

Figure 4: Latency performance of the proposed scheme against benchmark schemes. 


\subsection{Latency}

The latency of the six caching schemes is shown in Fig. 4. The average latency of the proposed scheme is $75.78 \mathrm{~ms}$, which is just $1.2 \%$ higher than LCD $(74.85 \mathrm{~ms})$ and $1.8 \%$ lower than the third best scheme, ProbCache (77.13ms). Specifically, Fig. 4aparameter $\alpha$. In contrast, Fig. 4d-4f illustrate the oscillation of latency against the popularity parameter $\alpha$ with the certain network $C C$. Generally, when the network $C C$ increases, the latency is naturally decreased because more contents could be cached in the network. In addition, when the user preferences become concentrated ( $\alpha$ value is large), the latency of all the caching schemes is decreased as well.

In particular, among the selected caching schemes, our proposed scheme can achieve lower latency when the $\alpha$ value is greater than 1. In this case, the content requests are concentrated towards a specific range of content. Since the proposed scheme is utilising caching resources near the end-user's path, this will incur a performance benefit to other when the content popularity is concentrated for $\alpha$ values larger than 1 . This is illustrated in Fig. $4 \mathrm{c}$ when $\alpha$ is 1.15, and the proposed scheme outperforms the rest benchmarks. Nonetheless, when the user preferences are dispersing, i.e. $\alpha$ values is less than 1 , as seen in Figures $4 \mathrm{a}$ and $4 \mathrm{~b}$, the latency performance of the proposed scheme is significantly improved with increasing network $C C$ in comparison to the remaining schemes. Specifically, in Fig. 4a, when $\alpha=0.75$, the proposed scheme has large delay, around $145 \mathrm{~ms}$, when network $C C$ is lower than $1 \%$. When $C C$ is between $1 \% \leq C C<5 \%$, the proposed scheme can achieve lower delay than RAND, Random Bernoulli and CSM, reaching the performance of ProbCache, but not as low as that of

A potential disadvantage of the near-path attribute is that when the content popularity is disperse, instead of searching for available caches only on the delivery path, as LCD and ProbCache do, the near-path attribute will have to incrementally search every node within each tier until a cache hit. This is incurring an increased latency from the onset 


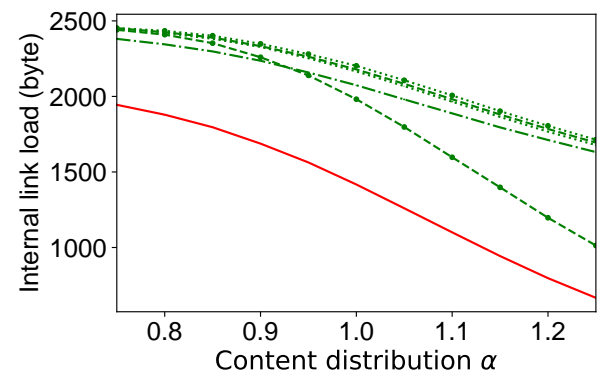

(a) $C C=0.2 \%$

....... ProbCache

....... RAND

$\rightarrow-$ CSM

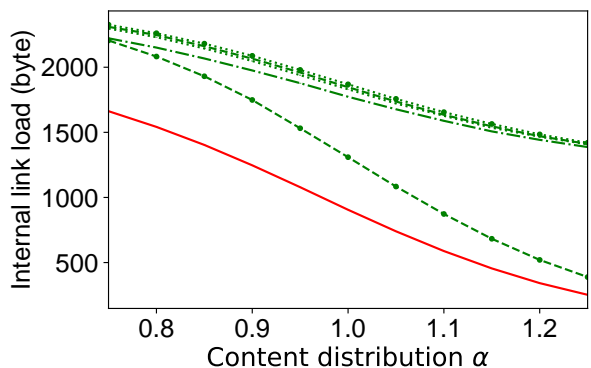

(b) $C C=5 \%$

- The Proposed Scheme

-.-. LCD

---- Random Bernoulli

Figure 5: Internal Link Load of the proposed scheme against benchmark schemes.

\subsection{Link Load \& Path Stretch}

Figures $5 \mathrm{a}$ and $5 \mathrm{~b}$ show the internal link load performance against a varying skewness parameter for a fixed value of $C C$ equal to $0.2 \%$ and $5 \%$, respectively. In both situations the proposed scheme performs significantly better compared to the five benchmarks. This a direct result of the full-time attribute that diverts the request traffic load outside the peak time period.

The average internal link load performance of the proposed scheme for all evaluated scenarios is 1084.72 bytes, which is $27.9 \%$ better than the second best scheme, CSM with an average of 1505.17 bytes. This reduction in link load happens due to the fact that the proposed scheme caches the content in the end-user devices. In particular, when the user preference is disperse (i.e. $\alpha=0.75$ in Fig. 5a), the internal link load of the proposed scheme is 1944.52 bytes and that of CSM is 2440.97 bytes. This results in a $20.3 \%$ improvement for the proposed scheme. When $\alpha$ increases to 1.25 , the internal link load of the proposed scheme is 606.97 bytes and that of CSM is 1012.89 bytes, which results in a $40.1 \%$ improvement for the proposed scheme. Similarly, when $C C=$ 5\% (Fig. 5b), the internal link load improvement against CSM ranges from 24.6\% up to $35.1 \%$.

The internal link load of ProbCache, RAND, Random Bernoulli and LCD greatly 


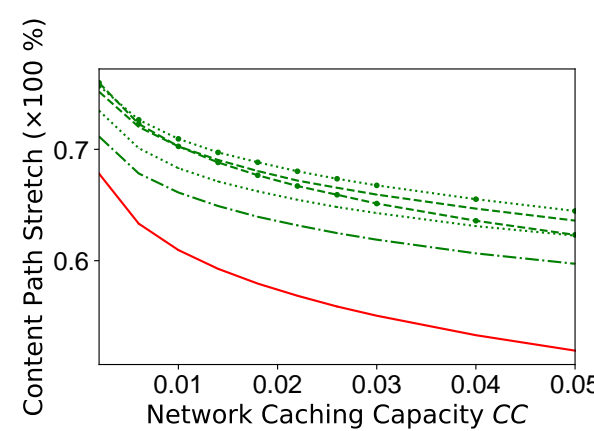

(a) $\alpha=0.85$

........ ProbCache

$\cdots *$ RAND

$\rightarrow-$ CSM

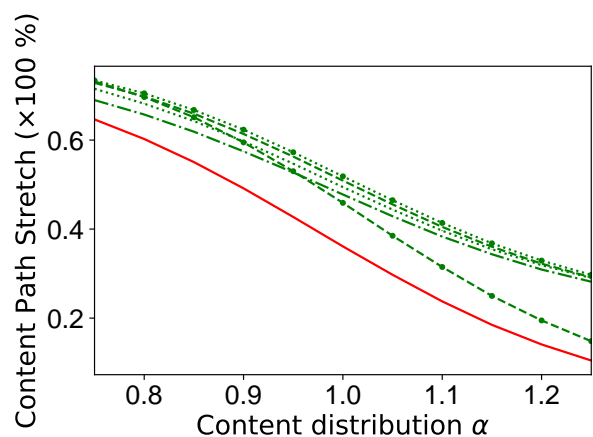

(b) $C C=3 \%$

- The Proposed Scheme
---. LCD
---- Random Bernoulli

Figure 6: Path Stretch indicates a significant gain for the proposed scheme in comparison to benchmarks.

under-perform in the majority of the evaluated scenarios. In most scenarios, CSM achieves a lower link load than LCD. Interestingly, when both $\alpha$ is less than 1 and $C C$ around $0.2 \%$, LCD can attain slightly lower internal link load than CSM, as seen in Fig. 5a. This happens because, when the user preferences are dispersing, the end-user device cannot cache the highly requested content within a limited caching capacity.

Finally, the experimental results for path stretch against varying $C C$ and $\alpha$ are shown at the left and right sides of Fig. 6 respectively. These are two representative examples from all 110 simulations and clearly highlight that the proposed scheme can significantly reduce the path stretch, due to the near-path approach. The reason for this is that when popular content is re-hit, it is gradually moved from higher to lower tiers, closer to the end-users. Thus, the delivery path of content is drastically reduced.

As shown in Fig. 6a as $C C$ increases from $0.2 \%$ to $5 \%$, the reduction of PS achieved by the proposed scheme ranges from $3.3 \%$ up to $7.3 \%$ in comparison to the second best scheme, LCD. In addition, as shown in Fig. 6b when $\alpha$ increases from 0.75 to 1.25 , the path stretch of the proposed scheme ranges from $8.6 \%$ to $4.3 \%$ in comparison to this case's second best scheme, CSM. 


\section{Conclusions and Future work}

This paper identifies six important design drivers of conventional ICN schemes by critically examining the state-of-the-art. After a thorough consideration of the six design drivers, a novel Fog caching scheme is proposed to efficiently facilitate content delivery in IoT environments. The proposed scheme is enabled both by the in-network caching capability of ICN, but also of end-user devices.

The proposed scheme leverages available information on node capabilities, such as forwarding, peer caching and content generation characteristics, as well as the tier-based locality within the Fog topology. By doing so, the scheme is able to construct adjustable sizes of clusters that efficiently provision caching within a Fog network. The caching nodes in the same Fog-Cluster work collaboratively to serve a group of end-users, that compose their respective user clusters.

In addition, this work introduces the near-path and full-time caching concepts. The former is an approach inspired by the on-path/off-path schemes, whilst the latter is a carefully designed hybrid between the reactive/proactive caching schemes. Specifically, the Fog caching nodes near the delivery path are identified that may efficiently reduce the content delivery latency and traffic load, as well as increase the cache hit ratio in Fog networks. Compared to traditional on-path/off-path schemes, the unique nearpath design is more suitable to IoT scenarios in which the data are mainly generated and requested at the network edge. In addition, the full-time feature performs both a reactive and proactive caching, only if the requested content is found at the original source, otherwise it only performs proactive caching to avoid creating congestion during the peak-time.

Thus, by incorporating three key attributes: flexible and adjustable node clustering, near-path content allocation and full-time caching, the six design drivers are satisfied. Experiments are conducted using a bespoke Fog topology running in the Icarus simulation environment, where content is located in both the core network, as well as, in end-user nodes. The experiments show that the proposed scheme design can achieve outstanding performance with this mixture of content generation. The proposed scheme is implemented and evaluated against Edge Caching, LCE, CL4M, CSM, ProbCache, 
LCD, RAND and Random Bernoulli caching schemes.

The experimental results clearly show that the cache hit ratio performance of the proposed scheme is always better than the benchmarks, especially when the network caching capacity increases towards $1 \%$. The latency performance of Fog caching is affected both by the content popularity and the caching capacity. In fact, the proposed scheme achieves lower latency compared to most popular caching schemes when the user preference dispersion, $\alpha$, is less than 1\%). Finally, on average, in terms of internal link load and path stretch, the proposed scheme achieves an improvement of $27.9 \%$ and $8.5 \%$, respectively.

In this work, user-devices and Fog caching nodes are organised into clusters depending on their capabilities and topological position. In future work, further criteria, such as content preference of users, geographical distribution and caching capacity, are expected to be considered during a node's cluster assignment. In addition, real-time design adjustments may be investigated depending on the network conditions and context. Finally, larger scale of experiments could be further considered.

\section{References}

[1] A. Kobusińska, C. Leung, C.-H. Hsu, S. Raghavendra, V. Chang, Emerging trends, issues and challenges in internet of things, big data and cloud computing, Future Generation Computer Systems 87 (2018) 416-419.

[2] A. Čolaković, M. Hadžialić, Internet of Things (IoT): A review of enabling technologies, challenges, and open research issues, Computer Networks 144 (2018) $17-39$.

[3] B. Nour, K. Sharif, F. Li, Y. Wang, Security and privacy challenges in informationcentric wireless internet of things networks, IEEE Security \& Privacy (2019) 1-1.

[4] S. Vural, P. Navaratnam, N. Wang, C. Wang, L. Dong, R. Tafazolli, In-network caching of internet-of-things data, in: IEEE International Conference on Communications (ICC), IEEE, 2014, pp. 3185-3190. 
[5] B. Nour, K. Sharif, F. Li, S. Biswas, H. Moungla, M. Guizani, Y. Wang, A survey of internet of things communication using ICN: A use case perspective, Computer Communications 142-143 (2019) 95-123.

[6] S. M. Oteafy, H. S. Hassanein, IoT in the fog: A roadmap for data-centric IoT Development, IEEE Communications Magazine 56 (3) (2018) 157-163.

[7] E.-K. Lee, J.-H. Lim, M. Gerla, Polycast: A new paradigm for informationcentric data delivery in heterogeneous mobile fog networks, International Journal of Distributed Sensor Networks 13 (9) (2017) 1550147717731529.

[8] D. Nguyen, Z. Shen, J. Jin, A. Tagami, ICN-fog: An information-centric fogto-fog architecture for data communications, in: IEEE Global Communications Conference (GLOBECOM), IEEE, 2017, pp. 1-6.

[9] I. Abdullahi, S. Arif, S. Hassan, Survey on caching approaches in information centric networking, Journal of Network and Computer Applications 56 (2015) 48-59.

[10] N. Laoutaris, H. Che, I. Stavrakakis, The LCD interconnection of LRU caches and its analysis, Performance Evaluation 63 (7) (2006) 609-634.

[11] L. Saino, I. Psaras, G. Pavlou, Icarus: A caching simulator for information centric networking (ICN), in: International ICST Conference on Simulation Tools and Techniques, ICST, 2014, pp. 66-75.

[12] L. Saino, I. Psaras, G. Pavlou, Understanding sharded caching systems, in: IEEE International Conference on Computer Communications (INFOCOM), IEEE, 2016, pp. 1-9.

[13] W. K. Chai, D. He, I. Psaras, G. Pavlou, Cache "less for more" in informationcentric networks (extended version), Computer Communications 36 (7) (2013) 758-770.

[14] I. Psaras, W. K. Chai, G. Pavlou, Probabilistic in-network caching for informationcentric networks, in: ICN workshop on Information-centric networking (ICN'12), ACM, 2012, pp. 55-60. 
[15] S. Arshad, M. A. Azam, M. H. Rehmani, J. Loo, Recent advances in informationcentric networking-based internet of things (ICN-IoT), IEEE Internet of Things Journal 6 (2) (2018) 2128-2158.

[16] O. Ascigil, S. Reñé, G. Xylomenos, I. Psaras, G. Pavlou, A keyword-based ICNIoT platform, in: ACM Conference on Information-Centric Networking (ICN'17), ACM, 2017, pp. 22-28.

[17] H. Khelifi, S. Luo, B. Nour, A. Sellami, H. Moungla, S. H. Ahmed, M. Guizani, Bringing deep learning at the edge of information-centric internet of things, IEEE Communications Letters 23 (1) (2018) 52-55.

[18] D. R. Cheriton, M. Gritter, TRIAD: A new next-generation Internet architecture.

[21] B. Nour, K. Sharif, F. Li, H. Moungla, Y. Liu, A unified hybrid information-centric naming scheme for IoT applications, Computer Communications 150 (2020) 103114.

[22] T. Koponen, M. Chawla, B.-G. Chun, A. Ermolinskiy, K. H. Kim, S. Shenker,

[19] G. Xylomenos, C. N. Ververidis, V. A. Siris, N. Fotiou, C. Tsilopoulos, X. Vasilakos, K. V. Katsaros, G. C. Polyzos, A survey of information-centric networking research, IEEE Communications Surveys \& Tutorials 16 (2) (2013) 1024-1049.

[20] L. Zhang, A. Afanasyev, J. Burke, V. Jacobson, K. Claffy, P. Crowley, C. Papadopoulos, L. Wang, B. Zhang, Named data networking, ACM SIGCOMM Computer Communication Review 44 (3) (2014) 66-73.

I. Stoica, A data-oriented (and beyond) network architecture, in: Proceedings of the 2007 conference on Applications, technologies, architectures, and protocols for computer communications, 2007, pp. 181-192.

[23] D. Trossen, M. J. Reed, J. Riihijärvi, M. Georgiades, N. Fotiou, G. Xylomenos, IP over ICN-the better IP?, in: 2015 European conference on networks and communications (EuCNC), IEEE, 2015, pp. 413-417. 
[24] M. F. Bari, S. R. Chowdhury, R. Ahmed, R. Boutaba, B. Mathieu, A survey of naming and routing in information-centric networks, IEEE Communications Magazine 50 (12) (2012) 44-53.

[25] S. Arianfar, P. Nikander, J. Ott, Packet-level caching for information-centric networking, in: ACM SIGCOMM, ReArch Workshop, 2010, pp. 1-16.

[26] K. Cho, M. Lee, K. Park, T. T. Kwon, Y. Choi, S. Pack, WAVE: Popularity-based and collaborative in-network caching for content-oriented networks, in: IEEE Conference on Computer Communications Workshops (INFOCOM WKSHPS), IEEE, 2012, pp. 316-321.

[27] Q. Wu, Z. Li, G. Xie, Codingcache: Multipath-aware ccn cache with network coding, in: Proceedings of the 3rd ACM SIGCOMM workshop on Informationcentric networking, 2013, pp. 41-42.

[28] H. Yan, D. Gao, W. Su, C. H. Foh, H. Zhang, A. V. Vasilakos, Caching strategy based on hierarchical cluster for named data networking, IEEE Access 5 (2017) 8433-8443.

[29] G. Ma, Z. Wang, M. Zhang, J. Ye, M. Chen, W. Zhu, Understanding performance of edge content caching for mobile video streaming, IEEE Journal on Selected Areas in Communications 35 (5) (2017) 1076-1089.

[30] D. Nguyen, K. Sugiyama, A. Tagami, Congestion price for cache management in information-centric networking, in: IEEE Conference on Computer Communications Workshops (INFOCOM WKSHPS), IEEE, 2015, pp. 287-292.

[31] D. Liu, B. Chen, C. Yang, A. F. Molisch, Caching at the wireless edge: Design aspects, challenges, and future directions, IEEE Communications Magazine 54 (9) (2016) 22-28.

760 [32] S. Wang, X. Huang, Y. Liu, R. Yu, CachinMobile: An energy-efficient users caching scheme for fog computing, in: IEEE/CIC International Conference on Communications in China (ICCC), IEEE, 2016, pp. 1-6. 
[33] Y. Hua, L. Guan, K. Kyriakopoulos, Semi-edge: From edge caching to hierarchical caching in network fog, in: International Workshop on Edge Systems, Analytics and Networking, ACM, 2018, pp. 43-48.

[34] D. Rossi, G. Rossini, On sizing CCN content stores by exploiting topological information, in: IEEE INFOCOM Workshops, IEEE, 2012, pp. 280-285.

[35] L. Saino, On the design of efficient caching systems, Ph.D. thesis, UCL (University College London) (2015).

[36] Y. Hayamizu, A. Nagata, M. Yamamoto, On-demand routing for chaining multiple functions in ICN-based in-network processing, in: IEEE International Symposium on Local and Metropolitan Area Networks (LANMAN), IEEE, 2018, pp. 7-12.

[37] N. Abani, T. Braun, M. Gerla, Proactive caching with mobility prediction under uncertainty in information-centric networks, in: ACM Conference on InformationCentric Networking (ICN'17), ACM, 2017, pp. 88-97.

[38] L. A. Belady, A study of replacement algorithms for a virtual-storage computer, IBM Systems journal 5 (2) (1966) 78-101.

[39] M. Garetto, E. Leonardi, V. Martina, A unified approach to the performance analysis of caching systems, ACM Transactions on Modeling and Performance Evaluation of Computing Systems (TOMPECS) 1 (3) (2016) 1-28.

[40] R. Zhang, J. Liu, T. Huang, R. Xie, Popularity based probabilistic caching strategy design for named data networking, in: IEEE Conference on Computer Communications Workshops (INFOCOM WKSHPS), IEEE, 2017, pp. 476-481.

[41] Z. Ming, M. Xu, D. Wang, Age-based cooperative caching in information-centric networking, in: International Conference on Computer Communication and Networks (ICCCN), IEEE, 2014, pp. 1-8.

[42] R. Schollmeier, A definition of peer-to-peer networking for the classification of peer-to-peer architectures and applications, in: Proceedings First International Conference on Peer-to-Peer Computing, IEEE, 2001, pp. 101-102. 
[43] A. Detti, L. Bracciale, P. Loreti, N. B. Melazzi, Modeling LRU cache with invalidation, Computer Networks 134 (2018) 55-65.

[44] N. K. Panigrahy, J. Li, D. Towsley, Network cache design under stationary requests: Exact analysis and poisson approximation, in: IEEE International Symposium on Modeling, Analysis, and Simulation of Computer and Telecommunication Systems (MASCOTS), IEEE, 2018, pp. 251-263.

[45] J. Liu, B. Bai, J. Zhang, K. B. Letaief, Cache placement in Fog-RANs: From centralized to distributed algorithms, IEEE Transactions on Wireless Communications 16 (11) (2017) 7039-7051.

[46] S. M. Azimi, O. Simeone, A. Sengupta, R. Tandon, Online edge caching and wireless delivery in fog-aided networks with dynamic content popularity, IEEE Journal on Selected Areas in Communications 36 (6) (2018) 1189-1202.

[47] Q. Yang, H. Deng, L. Wang, Y. Sheng, An almost-zero latency lightweight mechanism for caching decision in ICN content router, in: 2019 IEEE 38th International Performance Computing and Communications Conference (IPCCC), IEEE, 2019, pp. $1-8$.

[48] D. Man, Q. Lu, Y. Wang, Y. Wu, X. Du, M. Guizani, An adaptive cache management approach in ICN with pre-filter queues, Computer Communications 153 (2020) 250-263. 